these higher fuel prices - for example, through people driving less, power generators switching to cleaner fuels such as those from renewable energy sources, and households and businesses adopting energy-saving technologies. Because these responses are inherently uncertain, Jewell et al. used five different models to assess the consequences of subsidy reform. These models compared projections of fuel use and $\mathrm{CO}_{2}$ emissions with and without subsidy reform by region or country, using diverse assumptions about future economic growth, technological trends, energy prices and so on.

The authors found that removing all fossilfuel subsidies would have a limited impact on global energy demand by 2030 (a reduction of about 1-4\%). In addition, the share of energy from renewable sources would rise by less than $2 \%$, and global $\mathrm{CO}_{2}$ emissions would fall by only $1-4 \%$ (under both low and high oil prices). Consequently, in most regions, the $\mathrm{CO}_{2}$ reduction from subsidy reform would fall far short of what is needed to meet the Paris climate pledges (Fig. 1). The exceptions are regions such as Russia, the Middle East and North Africa, where subsidies are heavily concentrated and pledges are less ambitious.

There are two main reasons for the generally modest impact of subsidy reform on $\mathrm{CO}_{2}$ emissions. The first is that coal (the fossil fuel that emits by far the most $\mathrm{CO}_{2}$ per unit of energy) currently receives little subsidy. Instead, $60 \%$ of subsidies are for oil, and the remainder is largely for natural gas and for the electricity generated from fuels (see Figure $2 \mathrm{a}$ of the paper $\left.{ }^{1}\right)$. The second reason is that global subsidies have declined sharply, from US $\$ 570$ billion in 2013 to $\$ 330$ billion in 2015 .

However, I think that reform of fossil-fuel prices needs to go well beyond aligning them with production costs. Fuel prices should also reflect the consequences of their use for global warming and other environmental considerations, such as the costs of deaths resulting from air pollution and, in the case of road fuels, traffic congestion and accidents. Furthermore, prices for fuels purchased by households should include the general sales or value-added taxes that are applied to other consumer products.

A study ${ }^{2}$ in 2017 estimated that if fossil-fuel subsidies had been defined more broadly to reflect undercharging for environmental costs and general taxes, as well as production costs, these subsidies would have totalled $\$ 5.3$ trillion in 2015 (6.5\% of global gross domestic product). Furthermore, the study suggested that if prices had fully accounted for production costs, global and domestic environmental impacts and general taxes in 2013, global $\mathrm{CO}_{2}$ emissions would have been $21 \%$ lower than they were, air-pollution deaths associated with fossil fuels would have been 55\% lower, and government revenues as a percentage of gross domestic product would have been $4 \%$ higher.

Broader reform of fossil-fuel prices is

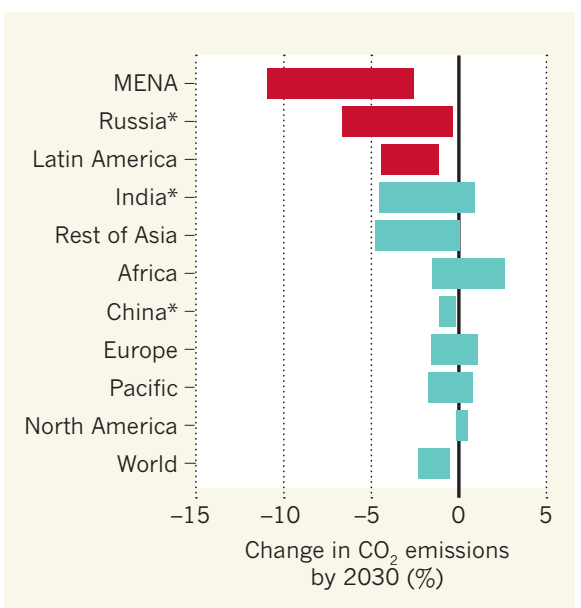

Figure 1 | Impact of fossil-fuel-subsidy reform. Fossil fuels are subsidized in many countries, and it was thought that removing these subsidies would lead to a substantial reduction in carbon dioxide emissions. However, Jewell et al. ${ }^{1}$ report an analysis suggesting that the resulting change in $\mathrm{CO}_{2}$ emissions by 2030 would be modest. The exceptions are regions in which current subsidies are heavily concentrated (shown in red), such as the Middle East and North Africa (MENA). The bars denote the range of emission changes predicted (under low oil prices), and asterisks indicate regions that constitute more than the designated country. (Adapted from Fig. 3 b of ref. 1.)

therefore urgent for both developed and developing countries. However, such a reform must be carefully crafted to enhance the prospects for success. A comprehensive plan should be developed, in consultation with stakeholders, that has clear goals and timetables. It should specify the taxes to be cut or the public investment programmes to be expanded, using revenue raised by fuel-price reform. In addition, there should be measures to compensate lowincome households for the effects of higher energy prices and to help workers who might lose their jobs in energy-intensive industries.

Researchers and international organizations (such as the International Monetary Fund, World Bank and the Organisation for Economic Co-operation and Development) have an important role in providing information and guidance to help policymakers drive forward subsidy reform and communicate the case for reform to the public. The information required includes the fossil-fuel prices that countries should adopt, both to meet their Paris climate pledges and to reflect the broader environmental costs.

But it also includes the effect of reform on energy systems, the economy, fiscal balances and vulnerable groups, and the trade-offs between higher fuel prices and other policy approaches, such as requirements for energy efficiency and renewable fuels. Analysis of ongoing reform experiences in different countries could also help governments to navigate around the political obstacles.

Rigorous studies, such as that by Jewell and colleagues, are essential. But there is a need to focus these studies on the broader reform issues discussed here, for which the stakes are especially high. .

Ian Parry is in the Fiscal Affairs Department, International Monetary Fund,

Washington DC 20431, USA.

e-mail:iparry@imf.org

1. Jewell, J. et al. Nature 554, 229-232 (2018).

2. Coady, D., Parry, I., Sears, L. \& Shang, B. World Dev. 91, 11-27 (2017)

\title{
Evolutionary race as predators hunt prey
}

Remote-sensing data for wild animals such as lions reveal that predators and prey optimize manoeuvrability rather than speed during the hunt. SEE ARTICLE P.183

\section{ANDREW A. BIEWENER}

$\mathrm{T}$ The survival of predators and prey depends on their respective abilities to successfully chase food and escape capture, thereby exerting strong selective pressure on their running ability and behavioural strategies. Perhaps nowhere on Earth does this play out more dramatically than on the African savannah, where the fastest terrestrial predators chase their fleet-footed prey. Yet direct measures of the key factors driving this type of hunt performance in the wild are difficult to obtain. On page 183 , Wilson et al. ${ }^{1}$ report findings from their use of data-capturing collars to track the movement dynamics of wild animals in Botswana during hunts. The authors also conducted computer modelling of predator-prey interactions and carried out laboratory tests to assess the properties of the animals' muscles.

In recent years, the ability to use remotesensing devices under natural field 


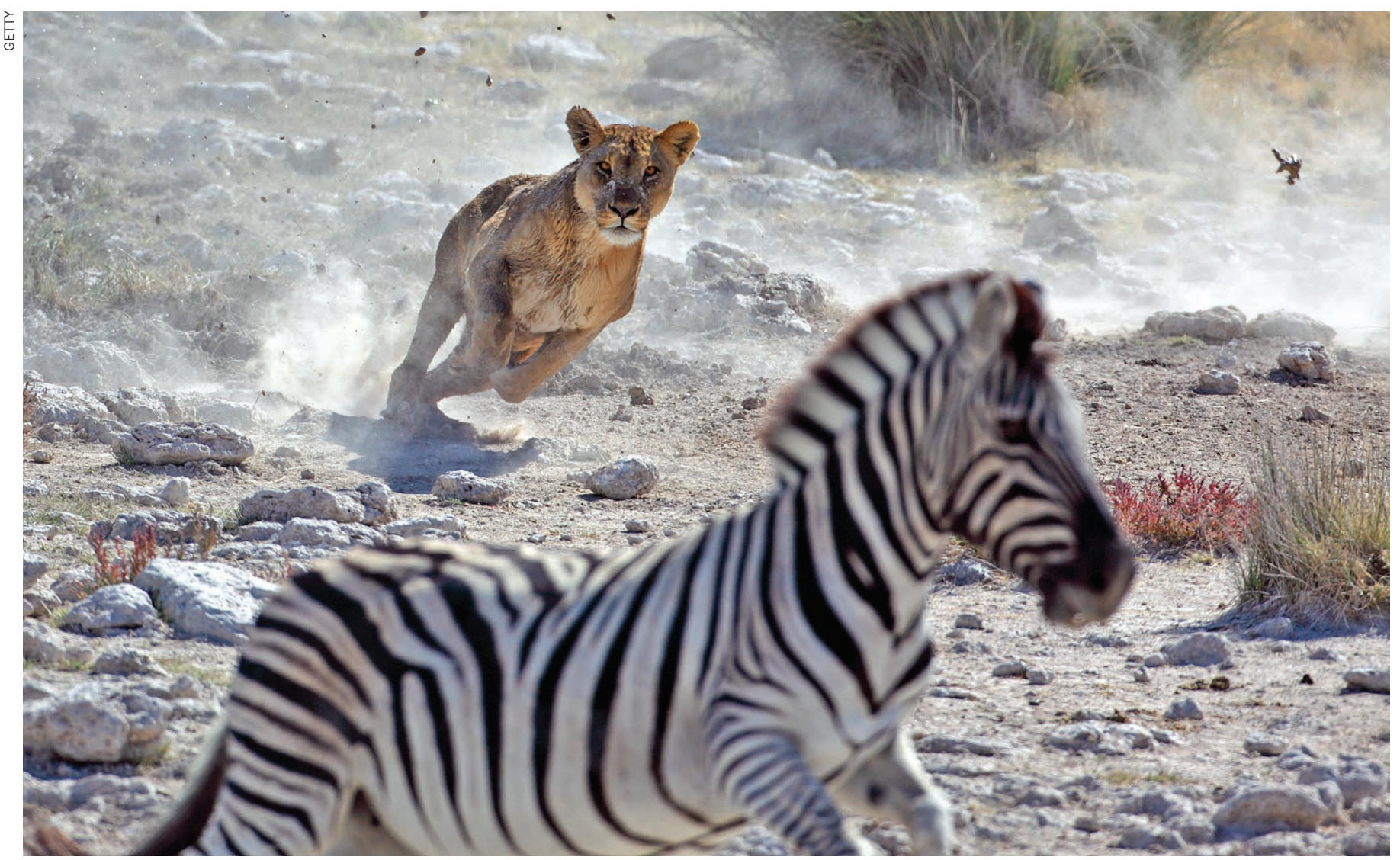

Figure 1 | A lioness hunting a zebra in Etosha National Park, Namibia. Wilson et al. ${ }^{1}$ report their analysis of the movement dynamics of predator-prey hunts in the wild in Africa using data gathered remotely from Global Positioning System sensing collars placed on lions, zebras, cheetahs and impala.

conditions and over long time frames has led many to study animals' migratory ${ }^{2,3}$, foraging ${ }^{4}$ and collective-movement behaviour ${ }^{5,6}$, which has provided fascinating insights into biomechanics, physiology and decision-making. Wilson and colleagues took a remote-sensing approach to study lions preying on zebras, and cheetahs preying on impala, in the wild. The authors temporarily immobilized animals and fitted them with lightweight collars containing technically sophisticated, customdesigned, miniature electronic and Global Positioning System (GPS) devices. The devices monitored the animals' location, movement direction and acceleration patterns. Wilson et al. tracked 9 lions, 5 cheetahs, 7 zebras and 7 impala, and recorded 2,726 high-speed runs for lions, 520 for cheetahs, 1,801 for zebras and 515 for impala. This remarkable data set logs individual animal strides and provides information about the speed, acceleration and turning performance of these predator-prey pairs.

The animals were not observed directly, and one limitation of the recorded data is that few, if any, of the movement tracks represented hunts between pairs of predator and prey, with both animals recorded as one hunts the other. Therefore, the hunting strategies of predator and prey must be inferred from the collar-recorded data, making the assumption that the movement patterns represent actual hunts. However, the locomotor performance recorded by the remote-sensing collars and the hunting strategies that could be inferred from these measurements are consistent with behavioural observations made by others ${ }^{7}$. Moreover, analysis of the full data set revealed that predators and prey exhibited manoeuvrability near the limits of their capability. Hence, although recordings of one-on-one hunts are lacking, the data were consistent with maximal predator-pursuit and prey-evasion performance, enabling the authors to model hunt outcomes.

After collar placement, a tiny biopsy of hindlimb muscle was taken from the animals for subsequent state-of-the-art laboratory testing of single-muscle-fibre contractility. This revealed that, compared with the muscle fibres sampled from the prey species, the predator muscle fibres deliver more power for a given muscle mass when they contract, allowing the predators to run faster and accelerate and decelerate more quickly than their prey. With more-powerful muscles than their prey and claws to grip the ground effectively, predators are better at accelerating into a turn (centripetal acceleration) than their prey are.

Wilson and colleagues' acceleration and GPS recordings indicated that, during inferred hunts, the predators and prey regularly achieved their maximal turning performance but ran at speeds well below their athletic capabilities. Running at speeds slower than maximum capacity during a pursuit enhances manoeuvrability, which improves the prey's probability of successful escape and enables predators to better track their prey's movements, thereby increasing the number of successful hunts.

Using their field-recorded locomotion data, Wilson and colleagues modelled predator and prey capture-evasion tactics to examine how different performance metrics, such as speed, separation distance between the animals, deceleration, acceleration and turning rate, would affect the outcome of a hunt. Evasion modelling showed that prey escape was more likely if a prey animal relied on turning more sharply and at areater rate than its pursuer. This type of behaviour increases the unpredictability of the prey's movement trajectory, as has also been observed for bipedal desert rodents fleeing a predator ${ }^{8}$. Wilson et al. noted that, during the predators' approach (Fig. 1), they exhibited greater deceleration and acceleration than that of the prey, allowing the predators to close in on and better track the prey's lateral movements. The close match of athletic performance between predators and prey highlights the strong selection pressure that has resulted in an evolutionary 'arms race' for improved locomotion ability in large carnivores and their large herbivorous prey.

The increasing use of remote-sensing technologies in animal studies is enabling the monitoring of factors such as animal 
acceleration, pressure (for example, during flight or when swimming at depth) and temperature. Such work promises to illuminate not only predator-prey interactions, but also how wild animals cope with other real-world issues $^{9,10}$. For example, this type of research could enhance our understanding of how animals are dealing with the impacts of climate change, or offer insight into the factors governing behaviours such as habitat selection, mating and foraging. Moreover, understanding how animals move might inspire the design of robots that can negotiate complex environments. -

Andrew A. Biewener is in the Department of Organismic and Evolutionary Biology, Harvard University, Massachusetts 01730, USA.

e-mail:abiewener@oeb.harvard.edu

1. Wilson, A. M. et al. Nature 554, 183-188 (2018).

2. Hawkes, L. A. et al. Proc. Natl Acad. Sci. USA 108, 9516-9519 (2011).

3. Portugal, S. J. et al. Nature 505, 399-402 (2014).

4. Tsoar, A. et al. Proc. Natl Acad. Sci. USA 108,
E718-E724 (2011).

5. Wittemyer, G., Getz, W. M., Vollrath, F. \& Douglas-Hamilton, I. Behav. Ecol. Sociobiol. 61, 1919-1931 (2007).

6. Nagy, M., Ákos, Z., Biro, D. \& Vicsek, T. Nature 464, 890-893 (2010).

7. Schaller, G. B. The Serengeti Lion: A Study of Predator-Prey Relations (Univ. Chicago Press, 1972).

8. Moore, T. Y., Cooper, K. L., Biewener, A. A. \& Vasudevan, R. Nature Commun. 8, 440 (2017).

9. Hussey, N. E. et al. Science 348, 1255642 (2015).

10.Kays, R., Crofoot, M. C., Jetz, W. \& Wikelski, M. Science $\mathbf{3 4 8}$, aaa2478 (2015).

This article was published online on 29 January 2018.

\section{A chirp, a roar and a whisper}

\section{In 2017, gravitational waves and electromagnetic radiation were detected from the merger of two stellar remnants called neutron stars. An observational analysis reveals how this radiation was released from the merger. SEE LETTER P.207}

\section{RALPH WIJERS}

$\mathrm{L}$ ast year, scientists reported the coalescence of two astronomical objects known as neutron stars ${ }^{1}$. The event, called GW170817, produced gravitational waves, which had weakened to a faint 'chirp' by the time they reached us. In addition, some of the matter in the neutron stars was ejected into space. Moments later, this matter was hit by a powerful jet of material from the merged stars, resulting in a roaring outburst of radiation at all wavelengths ${ }^{2}$. However, despite a flood of data, the process by which this radiation was generated has not been certain. On page 207,

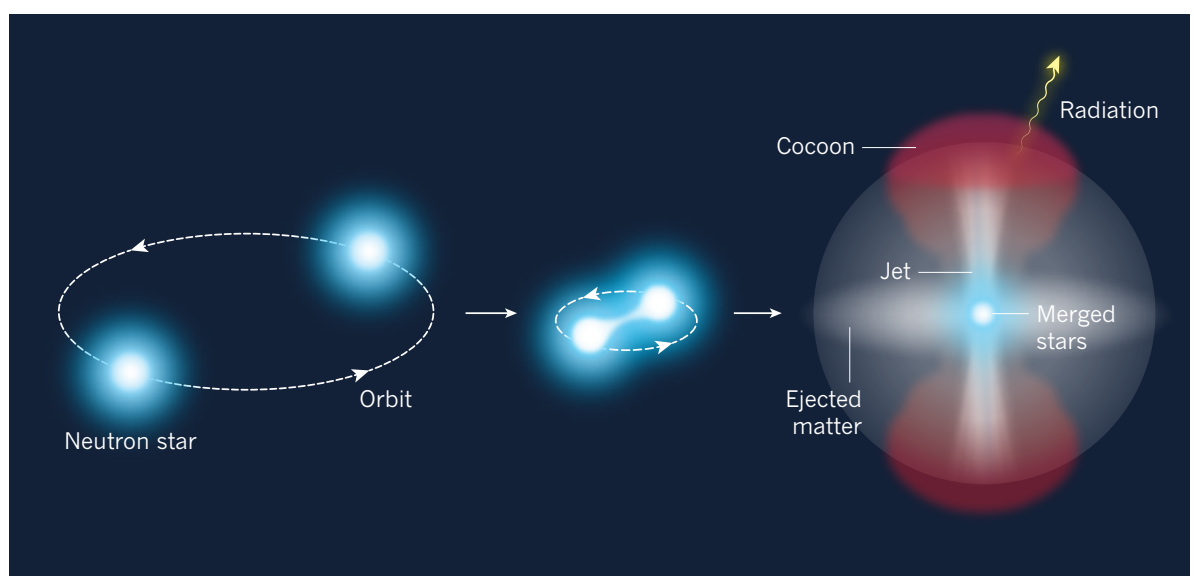

Figure 1 | Radiation from a neutron-star merger. A pair of stellar remnants called neutron stars can orbit each other, gradually getting closer, before eventually merging. In 2017, electromagnetic radiation was detected from a neutron-star merger ${ }^{2}$. Mooley et al. ${ }^{3}$ report evidence for a model that explains how this radiation was generated. In the model, some of the matter in the neutron stars is ejected. This matter is then energized by a powerful jet of material from the merged stars, creating a relatively slow-moving 'cocoon' of matter. The cocoon then emits the observed radiation. fields, nuclear reactions, particle acceleration and radiation come into play. Astronomers cannot create and tune experiments, but must make do with the messy ones performed by nature.

What astronomers can do, however, is take advantage of two of the biggest revolutions in the field since the invention of the telescope. First, in the twentieth century, astronomy became multi-wavelength: we can now detect radiation across the electromagnetic spectrum (from radio waves to $\gamma$-rays). Second, in this century, it became multi-messenger: we can now detect a broad range of emissions from high-energy cosmic rays and neutrinos to gravitational waves. The discovery of GW170817 demonstrated the full potential of these advances for the first time.

After being alerted to the gravitational-wave signal, astronomers used just about every type of telescope available to try to view the event. As a result, a wide variety of data was obtained, potentially providing enough information to pin down a complete picture of what physically happened when the neutron stars merged. In particular, NASA's Fermi Gamma-ray Space Telescope detected a flash of $\gamma$-rays that had formed within two seconds of the merger ${ }^{7}$. The properties of the flash were consistent with a $\gamma$-ray burst (a cosmic explosion long thought to be related to neutron-star mergers), which immediately increased interest in GW 170817. However, the exact cause of the $\gamma$-ray emission became a matter of debate.

Standard $\gamma$-ray bursts can be produced only by a jet - an outflow of material moving at a speed at least $99.9 \%$ that of light. But the burst from GW170817 was about 10,000 times weaker than these bursts and seen only because it occurred relatively close to us ${ }^{7}$. Such a weak burst could have come from an off-axis jet (one that was aimed away from us), which would allow only the tiny fraction of light that it emitted sideways to be observed. But it could also have been produced by a comparatively slowmoving cocoon of matter, perhaps travelling at 'only' 95\% of the speed of light (Fig. 1).

The initial papers ${ }^{2,8}$ concluded that both scenarios are possible, and that additional data should allow us to identify which one is correct. Mooley et al. now fulfil this promise. They show that although the outburst of radiation 